Supporting Information for

\title{
Design principles for sensitivity optimization in plasmonic hydrogen sensors
}

Florian Sterl*, Nikolai Strohfeldt, Steffen Both, Ediz Herkert, Thomas Weiss, and Harald Giessen

$4^{\text {th }}$ Physics Institute and Research Center SCoPE, University of Stuttgart, Pfaffenwaldring 57, 70569 Stuttgart, Germany 


\section{Derivation of the signal-to-noise ratio (SNR)}

In the following, we will provide an analysis of the origin of the signal-to-noise ratio in the optical signal of a single-wavelength optical hydrogen sensor, as described in the main manuscript. The plasmonic sensor substrate in such a sensor has a certain reflectance $R\left(\lambda, p_{H 2}\right)$. Upon exposure to hydrogen gas the absorption of hydrogen by the palladium nanoparticles leads to a change in $R$, giving an absolute difference in reflectance of

$$
\Delta R_{a b s}=R\left(p_{H 2}\right)-R_{0}
$$

where $R_{0}$ is the reflectance in the absence of hydrogen: $R_{0}=R\left(p_{H 2}=0\right)$.

At the same time, we can define a relative change in reflectance:

$$
\Delta R_{r e l}=\frac{R\left(p_{H 2}\right)-R_{0}}{R_{0}}
$$

The central question is: which of these two quantities do we have to optimize in order to minimize the signal-to-noise ratio of our detector?

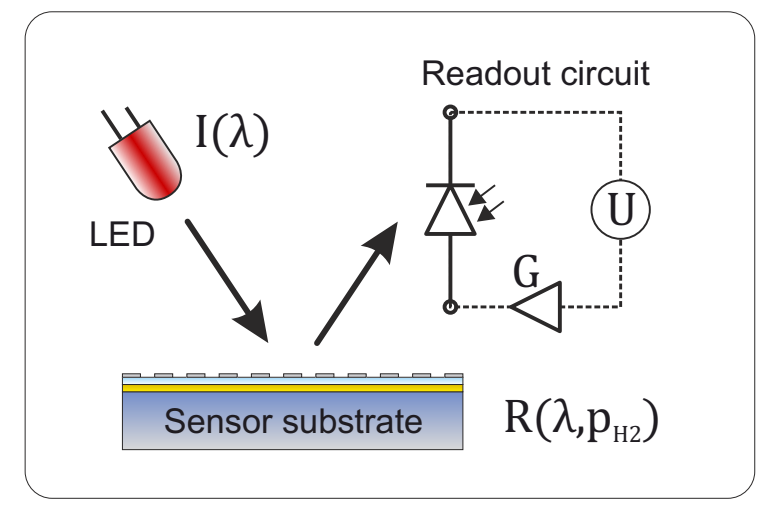

Figure S1: Sensor with LED, reflective sensor substrate and photodiode with read-out circuitry.

Here, we consider a simple single-wavelength sensor that consists of an LED with intensity $I(\lambda)$, a sensor substrate with reflectance $R\left(\lambda, p_{H_{2}}\right)$, and a photodiode connected to electronic read-out circuit with gain $G$. This means that at any given time, the detected voltage $U$ is:

$$
U\left(p_{H 2}, \lambda\right)=I(\lambda) \cdot R\left(\lambda, p_{H 2}\right) \cdot G
$$

Each of these three quantities will have some level of noise. In a first-order approximation, this noise is independent of the actual value of each quantity. Inserting these signal noises (and omitting the notation of $\lambda$ and $p_{H_{2}}$ for the sake of readability), we obtain the measured voltage plus noise:

$$
U+\delta U=(I+\delta I) \cdot(R+\delta R) \cdot(G+\delta G)
$$


The noise level $\delta U$ can be estimated as follows:

$$
\delta U=\frac{d U}{d I} \delta I+\frac{d U}{d R} \delta R+\frac{d U}{d G} \delta G=R G \cdot \delta I+I G \cdot \delta R+I R \cdot \delta G,
$$

from which we can calculate the root-mean-square noise:

$$
\begin{aligned}
\sqrt{\left\langle\delta U^{2}\right\rangle} & =\sqrt{(R G\langle\delta I\rangle+I G\langle\delta R\rangle+I R\langle\delta G\rangle)^{2}} \\
& =\sqrt{(R G\langle\delta I\rangle+I G\langle\delta R\rangle+I R\langle\delta G\rangle) \cdot(R G\langle\delta I\rangle+I G\langle\delta R\rangle+I R\langle\delta G\rangle)} \\
& =\sqrt{R^{2} G^{2}\left\langle\delta I^{2}\right\rangle+I^{2} G^{2}\left\langle\delta R^{2}\right\rangle+I^{2} R^{2}\left\langle\delta G^{2}\right\rangle+2 I R G^{2}\langle\delta I \delta R\rangle+\ldots}
\end{aligned}
$$

In this last expression, there are three terms that contain products of different noise levels. As long as the three different noise sources are uncorrelated, they cancel out and the resulting mean is zero, so that these terms can be omitted:

$$
\sqrt{\left\langle\delta U^{2}\right\rangle}=\sqrt{R^{2} G^{2}\left\langle\delta I^{2}\right\rangle+I^{2} G^{2}\left\langle\delta R^{2}\right\rangle+I^{2} R^{2}\left\langle\delta G^{2}\right\rangle} .
$$

The signal $S$ that is obtained in the end as a function of the hydrogen concentration is the difference between the detected voltage and the zero-level voltage:

$$
S\left(p_{H 2}\right)=\Delta U=U\left(p_{H 2}\right)-U_{0} .
$$

In order to achieve a detectable signal at a certain hydrogen concentration, this signal must be bigger than the noise in $S$; in other words, the signal-to-noise ratio (SNR) must be bigger than one:

$$
\mathrm{SNR}=\frac{\mathrm{S}}{\sqrt{\left\langle\delta \mathrm{S}^{2}\right\rangle}}>1
$$

The signal noise $\delta S$ follows from error propagation (leaving out the root-mean-square notation):

$$
\delta S=\sqrt{\left(\delta U\left(p_{H 2}\right)\right)^{2}+\left(\delta U_{0}\right)^{2}} .
$$

If we assume that $\delta U$ as a function of $p_{H_{2}}$ is constant, this means that

$$
\sqrt{\left\langle\delta S^{2}\right\rangle}=\sqrt{2\left\langle\delta U^{2}\right\rangle} \text {. }
$$

Note: this is not entirely true. As equation S5 shows, the reflectance (and thus $p_{H 2}$ ) does influence the voltage noise $\delta U$. However, we are talking about very small changes in $R$, so that the assumption can be made that $\delta U(R)=\delta U\left(R_{0}\right)$ for any practical purpose.

Inserting equations S3 and S5 into equation S9, we can write:

$$
\mathrm{SNR}=\frac{\mathrm{IG} \cdot \Delta \mathrm{R}_{\mathrm{abs}}}{\sqrt{2\left(\mathrm{R}^{2} \mathrm{G}^{2}\left\langle\delta \mathrm{I}^{2}\right\rangle+\mathrm{I}^{2} \mathrm{G}^{2}\left\langle\delta \mathrm{R}^{2}\right\rangle+\mathrm{I}^{2} \mathrm{R}^{2}\left\langle\delta \mathrm{G}^{2}\right\rangle\right)}},
$$

which can be simplified: 


$$
\begin{aligned}
\mathrm{SNR} & =\frac{\Delta R_{a b s}}{\frac{1}{I G} \sqrt{2\left(R^{2} G^{2}\left\langle\delta I^{2}\right\rangle+I^{2} G^{2}\left\langle\delta R^{2}\right\rangle+I^{2} R^{2}\left\langle\delta G^{2}\right\rangle\right)}} \\
& =\frac{\Delta R_{a b s}}{\sqrt{2\left(\frac{R^{2} G^{2}}{I^{2} G^{2}}\left\langle\delta I^{2}\right\rangle+\frac{I^{2} G^{2}}{I^{2} G^{2}}\left\langle\delta R^{2}\right\rangle+\frac{I^{2} R^{2}}{I^{2} G^{2}}\left\langle\delta G^{2}\right\rangle\right)}} \\
& =\frac{\Delta R_{a b s}}{\sqrt{2 R^{2}\left(\frac{\left\langle\delta I^{2}\right\rangle}{I^{2}}+\frac{\left\langle\delta R^{2}\right\rangle}{R^{2}}+\frac{\left\langle\delta G^{2}\right\rangle}{G^{2}}\right)}}=\frac{\Delta R_{a b s}}{R \sqrt{2\left(\frac{\left\langle\delta I^{2}\right\rangle}{I^{2}}+\frac{\left\langle\delta R^{2}\right\rangle}{R^{2}}+\frac{\left\langle\delta G^{2}\right\rangle}{G^{2}}\right)}} .
\end{aligned}
$$

Now we can use equation $S 13$ to judge what we have to do in order to maximize the SNR, which is how we optimize the sensitivity of our detector.

There are, in fact, two distinct cases.

- The noise in the detector, meaning the LED, the photodiode and the read-out circuit, is prevalent, so that $\delta I$ and $\delta G$ (or even only one of them) is larger than the noise in the substrate reflectance $\delta R$ :

$$
\mathrm{SNR} \approx \frac{\Delta \mathrm{R}_{\mathrm{abs}}}{\mathrm{R} \sqrt{2\left(\frac{\left\langle\delta \mathrm{I}^{2}\right\rangle}{\mathrm{I}^{2}}+\frac{\left\langle\delta \mathrm{G}^{2}\right\rangle}{\mathrm{G}^{2}}\right)}} .
$$

Maximizing the SNR can in this case be done by maximizing $\Delta R_{a b s}$, but also by minimizing $R$. In this case, it could be beneficial to choose a sensor design which gives the lowest possible reflectance, even though this comes at the expense of a lower $\Delta R_{a b s}$ (see figure S2).

- The detector noise be effectively eliminated, for example by using a stabilized power supply for the LED and balance detection to read out the photodiode voltage. In this case, fluctuations of the substrate reflectance (due to any environmental factors such as the temperature, the gas flow rate, or aging of the substrate) play the most important role:

$$
\mathrm{SNR} \approx \frac{\Delta \mathrm{R}_{\mathrm{abs}}}{\mathrm{R} \sqrt{2 \frac{\left\langle\delta \mathrm{R}^{2}\right\rangle}{\mathrm{R}^{2}}}}=\frac{\Delta \mathrm{R}_{\mathrm{abs}}}{\sqrt{2 \cdot\left\langle\delta \mathrm{R}^{2}\right\rangle}},
$$

where $R$ drops out of the equation. We thus only want to maximize the absolute reflectance change $\Delta R_{a b s}$, while the value of $R_{0}$ does not make any difference.

Since one can safely assume that the second case is the more realistic one - achievable through careful engineering of the detector hardware and electronics - we learn from this that the primary aim should be to maximize $\Delta R_{a b s}$. Of course, if one has to choose between two sensor designs that provide the same $\Delta R_{a b s}$ at different reflectances $R_{0}$, one might argue that the lower $R_{0}$ is the better option, but this should not be the primary target. 


\section{Additional data}

In this section, we provide additional data from numerical calculations as well as experiments, as well as a description of the baseline correction method employed in the time-dependent reflectance measurements.

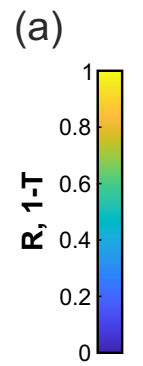

(b)
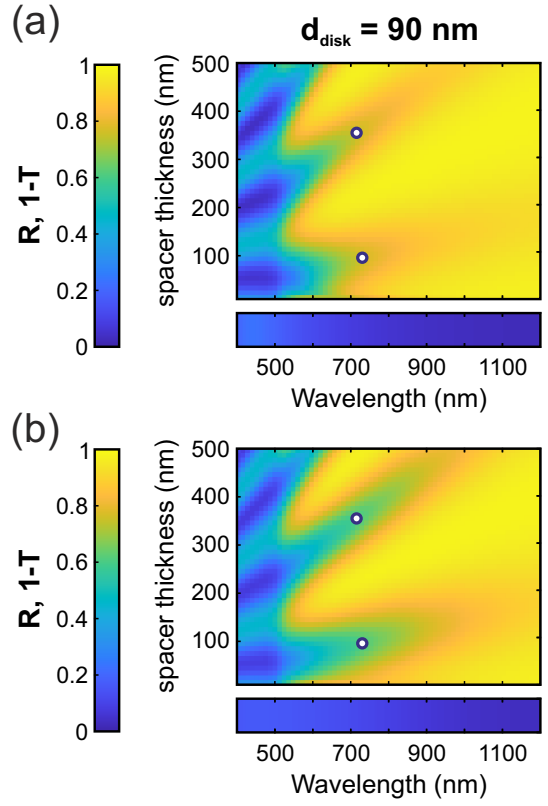

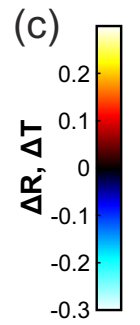
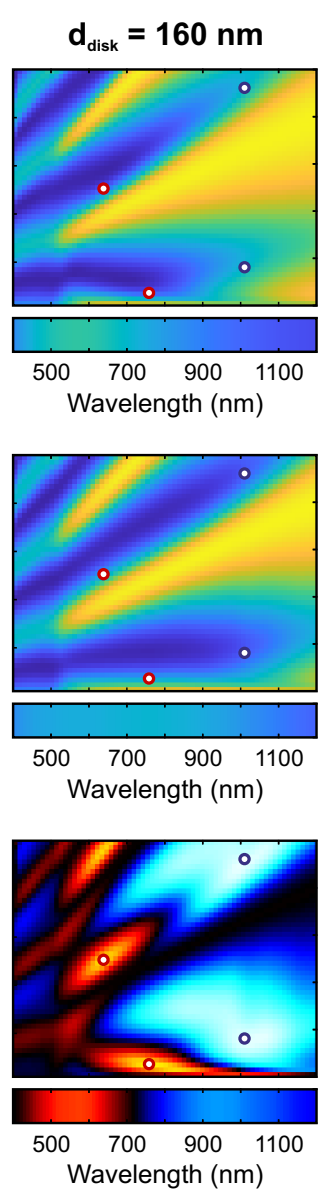
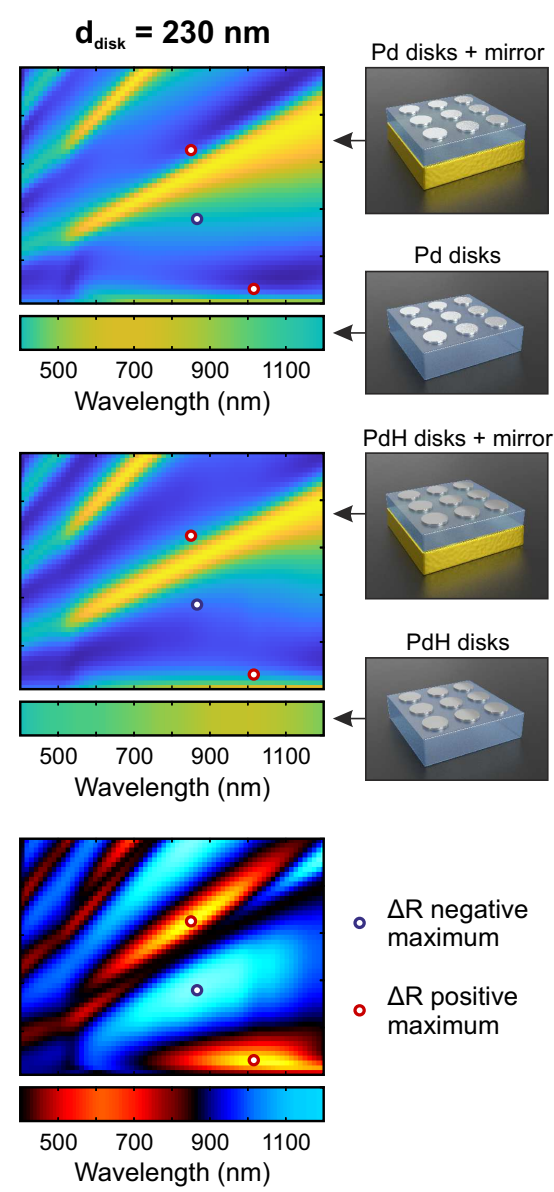

$\Delta \mathrm{R}$ negative maximum

- $\Delta \mathrm{R}$ positive maximum

Figure S2: (a) Calculated reflectance spectra as a function of spacer thickness and disk diameter, at a periodicity of $300 \mathrm{~nm}$. (b) Calculated reflectance spectra for the same structures as in (a), with PdH instead of Pd disks and taking into account a volume expansion of $10 \%$. (c) Plots of the resulting $\Delta R$ vs. spacer thickness and wavelength. The positions of some exemplary local maxima of $|\Delta R|$ are indicated. 

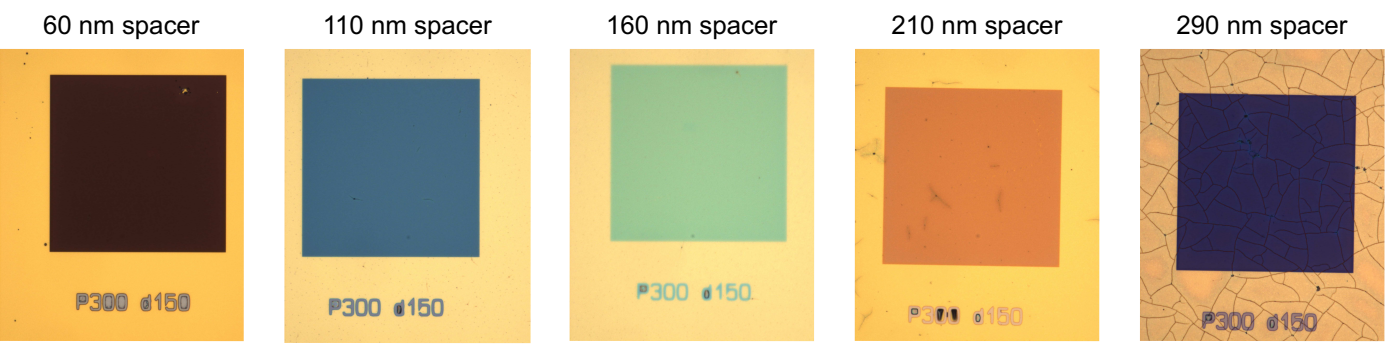

Figure S3: Examplary microscope images of the fabricated sensor structures with $P=300 \mathrm{~nm}$, $d_{\text {disk }}=202 \pm 7 \mathrm{~nm}$. The changing reflectance spectrum manifests itself in the visible color. In the thickest spacers, cracking of the $\mathrm{MgF}_{2}$ can be observed. Each field measures $100 \times 100$ microns.
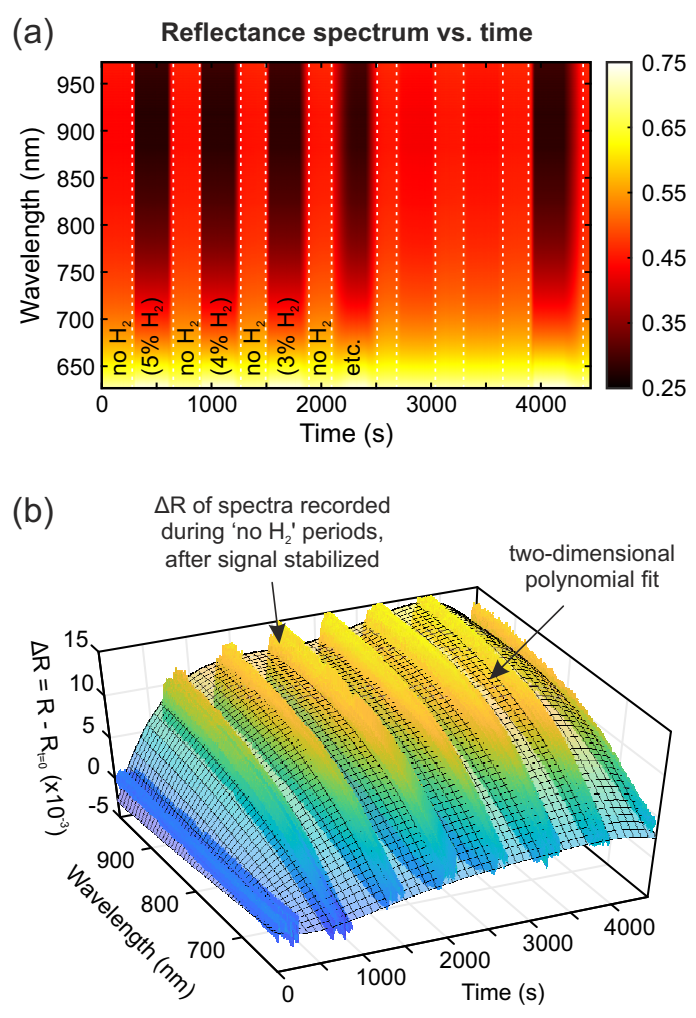
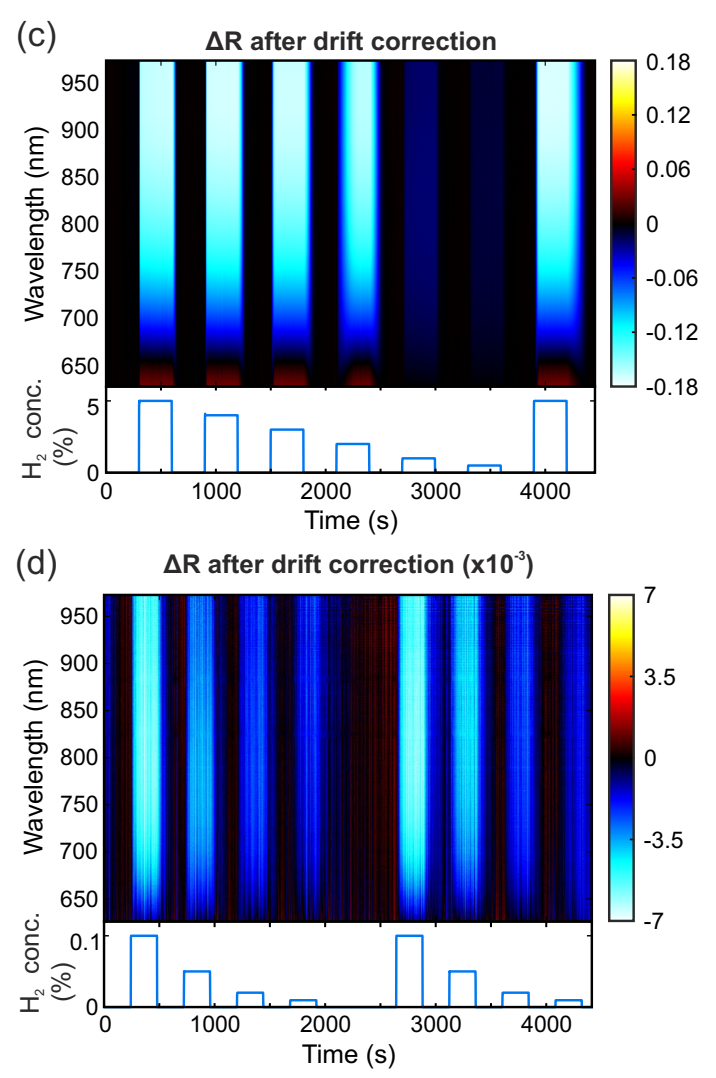

Figure S4: Example of the drift correction applied to the $R$ time trace for the structures with $t_{\text {spacer }}=160 \mathrm{~nm}$ and $d_{\text {disk }}=174 \pm 6 \mathrm{~nm}$ (a). First, the signal $\Delta R$ is calculated by subtracting $R_{0}$, obtained from averaging over the spectra recorded before the first gas exposure. Then a two-dimensional polynomial surface (degrees 5 and 3 along the time and wavelength axes, respectively) is fitted to the surface resulting from all spectra in between the hydrogen exposures, omitting the times at which the spectrum is still strongly changing during dehydrogenation (b). This polynomial surface is then subtracted from all $\Delta R$ spectra (c). After each measurement cycle, at least one extra hydrogen exposure is performed to verify the reproducibility of the signal. This is also shown in (d) for the low hydrogen concentrations for the same structure. 


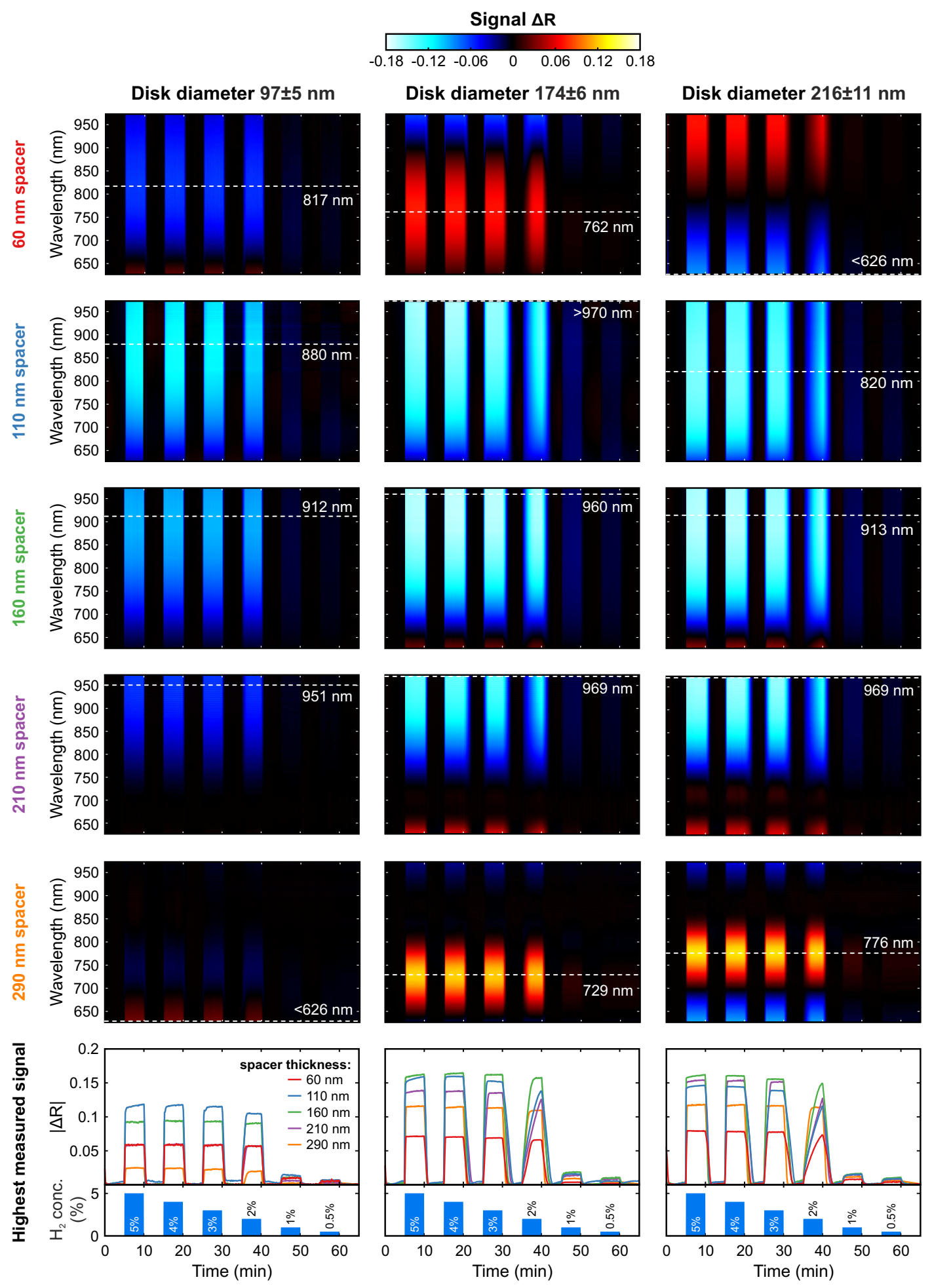

Figure S5: Measured reflectance differences vs. wavelength and time during hydrogen concentration cycles for the five different spacer thicknesses and three different disk diameters, with corresponding time traces of the maximum reflectance difference. 

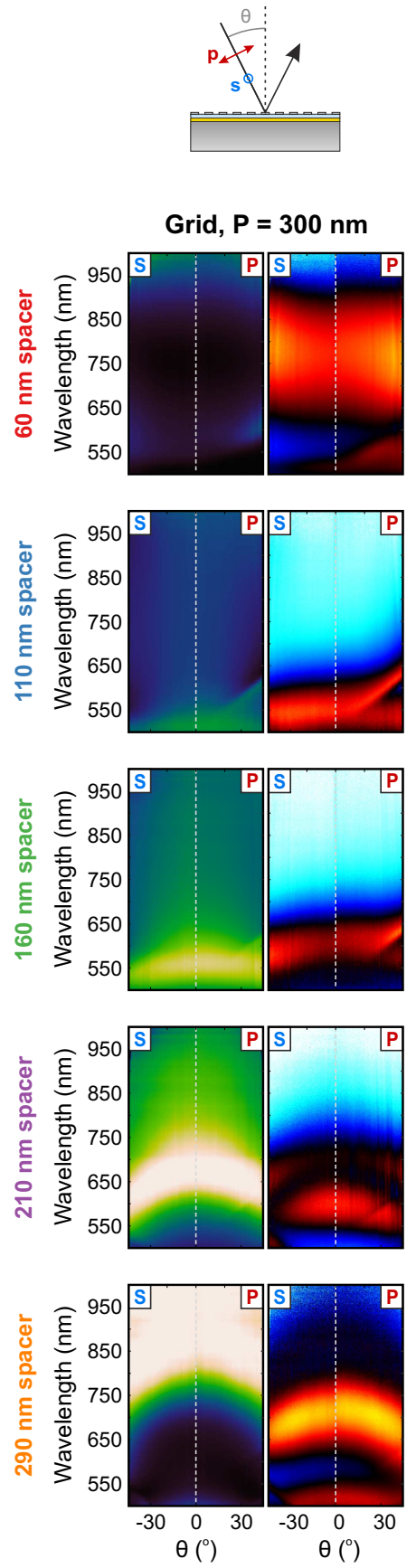

Reflectance in $\mathbf{N}_{\mathbf{2}}$

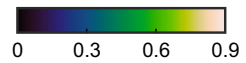

Disordered,

$\mathrm{S}_{\min }=250 \mathrm{~nm}$
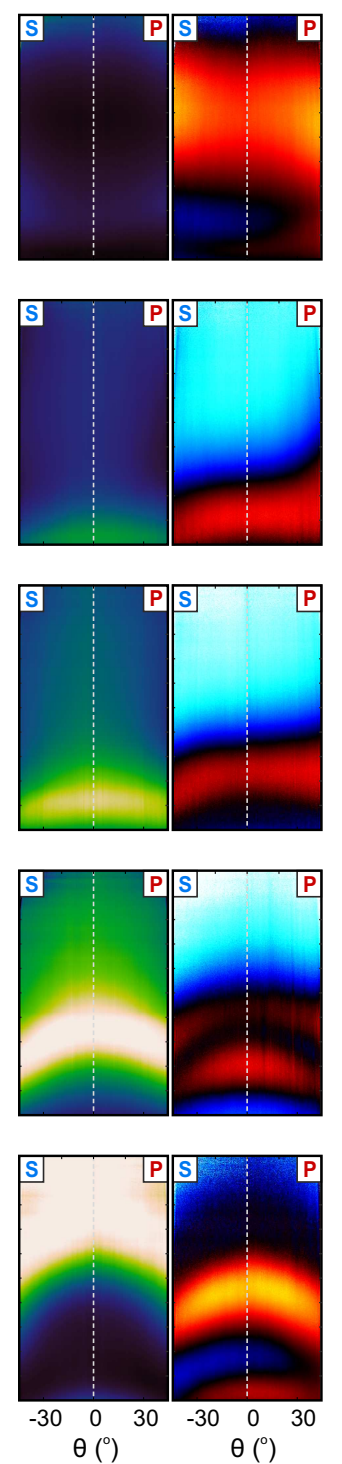

$\Delta R$ in $5 \% \mathrm{H}_{2}$
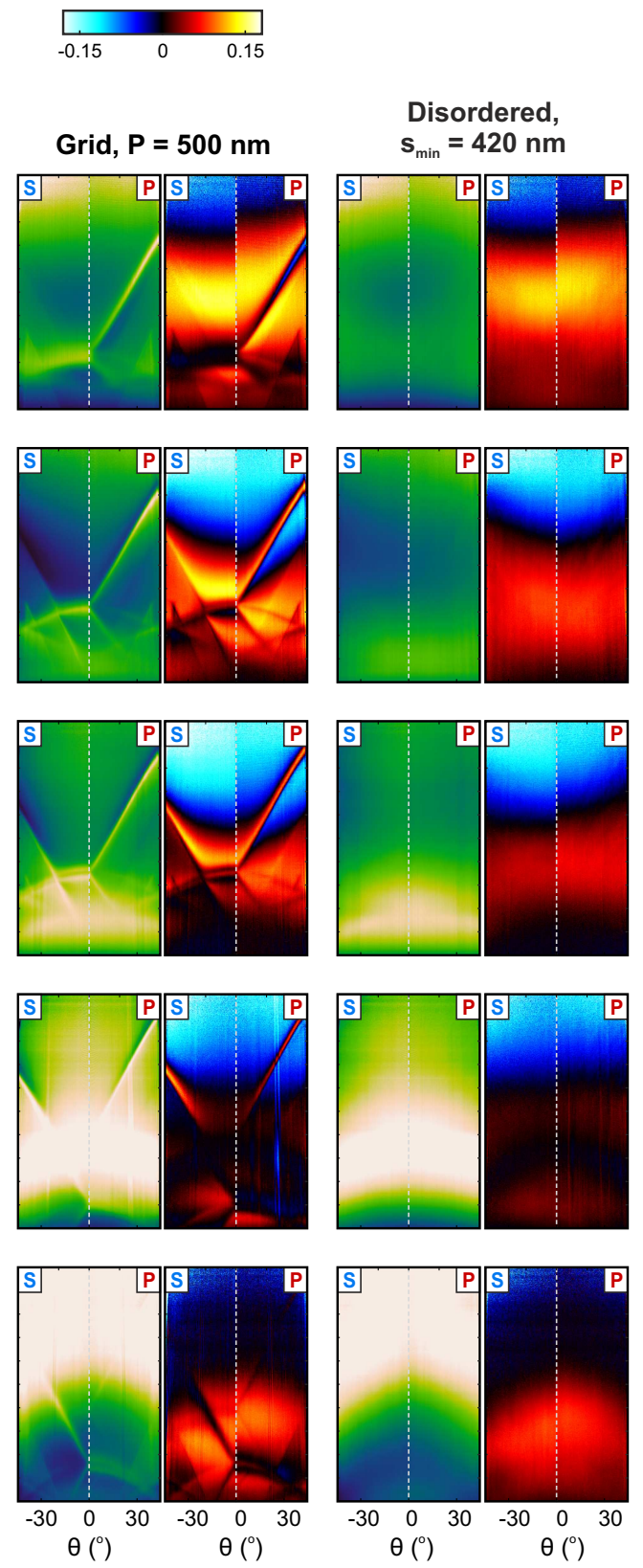

Figure S6: Overview of angle-dependent reflectance measurements for all fabricated spacer thicknesses, for the two different periodicities and corresponding disordered realizations, for $s$ and p polarization, alongside the obtained signal $\Delta R$ in $5 \% \mathrm{H}_{2}$. 


\section{Analysis of angle-dependent reflectance}

In the following, we provide a detailed analysis of the sharp angle- and polarization-dependent resonance features that occur in the measured reflectance spectra when the periodicity is chosen as large as $500 \mathrm{~nm}$ (measurements in Figure 7 of the main text and in Figure S6). Corresponding numerically calculated spectra are provided in Figure S7(a). The spectra show reflectance vs. wavelength and angle for a Pd absorber structures with $P=500 \mathrm{~nm}, d_{\text {disk }}=160 \mathrm{~nm}$, for the five different fabricated spacer thicknesses and were calculated with the scattering matrix approach mentioned in the main text. As it can be seen, the calculated spectra agree very well with the measured ones and exhibit the same resonance features.

Let us now investigate the origin of these features. For an incidence angle of $\theta=0^{\circ}$, the occurring resonance peaks are marked with red and blue dots in the calculated spectra. For small spacer thicknesses $(60 \mathrm{~nm}$ and $110 \mathrm{~nm}$ ), only one sharp peak is observed (red dots), while for thicknesses of $160 \mathrm{~nm}$ and larger, a second sharp peak arises (blue dots). With increasing spacer thickness, both peaks shift to longer wavelengths.

In Figure S7(b), we depict the corresponding electric field distributions (again derived with the scattering matrix approach). The top row corresponds to the spectral positions marked with the red dots. The fields can be associated with the excitation of a surface plasmon polariton (SPP) mode [1] that propagates along the interface between the gold mirror and the dielectric spacer. Note that for a spacer thickness of $210 \mathrm{~nm}$, no distinct SPP feature is visible in the field, since this resonance coincidentally overlaps with a reflection maximum of the perfect absorber (cf. Figure 2 of the main text), which dominates the optical response at this spectral position. Analyzing the orientations of the fields (not shown here) reveals that the mode exhibits transverse-magnetic (TM) polarization, as it is expected for such SPP modes. The bottom row corresponds to the spectral positions marked with the blue dots. The fields show the excitation of a waveguide mode [2] that propagates inside the spacer layer. Analyzing the field orientation (again not shown), reveals that the waveguide mode exhibits transverse-electric (TE) polarization.

The field distributions imply that the sharp resonances in the spectra can be attributed to SPP and waveguide modes. In order to validate this, let us now switch to a more sophisticated description. The system consists of multiple layers that are stacked in the $z$ direction (from bottom to top: substrate, gold mirror, spacer layer, Pd nanodisks, and air). We note that if we would remove the Pd nanodisks, the system would be completely homogeneous (i.e., translationally symmetric) in the $x y$ plane. Only the presence of the Pd nanodisks breaks this translational symmetry and introduces a periodicity in the $x$ and $y$ direction. In the following, we will refer to the system without the Pd nanodisks as the homogeneous system.

The SPP and the waveguide modes are eigenmodes of the homogeneous system. In general, the dispersion relation of such modes can be written as a function $\omega_{\mathrm{m}}\left(k_{\|}\right)$[3], where $k_{\|}$denotes the in-plane momentum (referring to the plane of propagation, i.e., the $x y$ plane) and $\omega_{\mathrm{m}}$ with $\mathrm{m}=\{\mathrm{SPP}$, waveguide\} denotes the eigenfrequency of the respective mode. In the above description, $k_{\|}$is a real number, while $\omega_{\mathrm{m}}$ is in general complex, with $\operatorname{Re}\left(\omega_{\mathrm{m}}\right)$ representing the resonance frequency of the mode and $-2 \operatorname{Im}\left(\omega_{\mathrm{m}}\right)$ specifying its linewidth [4]. The dispersion relations $\omega_{\mathrm{m}}\left(k_{\|}\right)$of the SPP and waveguide modes in the homogeneous system were numerically calculated by following the method provided in Refs. [5, 4]. Note that this method requires that the material parameters of the system can be evaluated at complex frequencies, which has been achieved by modeling the permittivity of the gold according to Ref. [6]. 

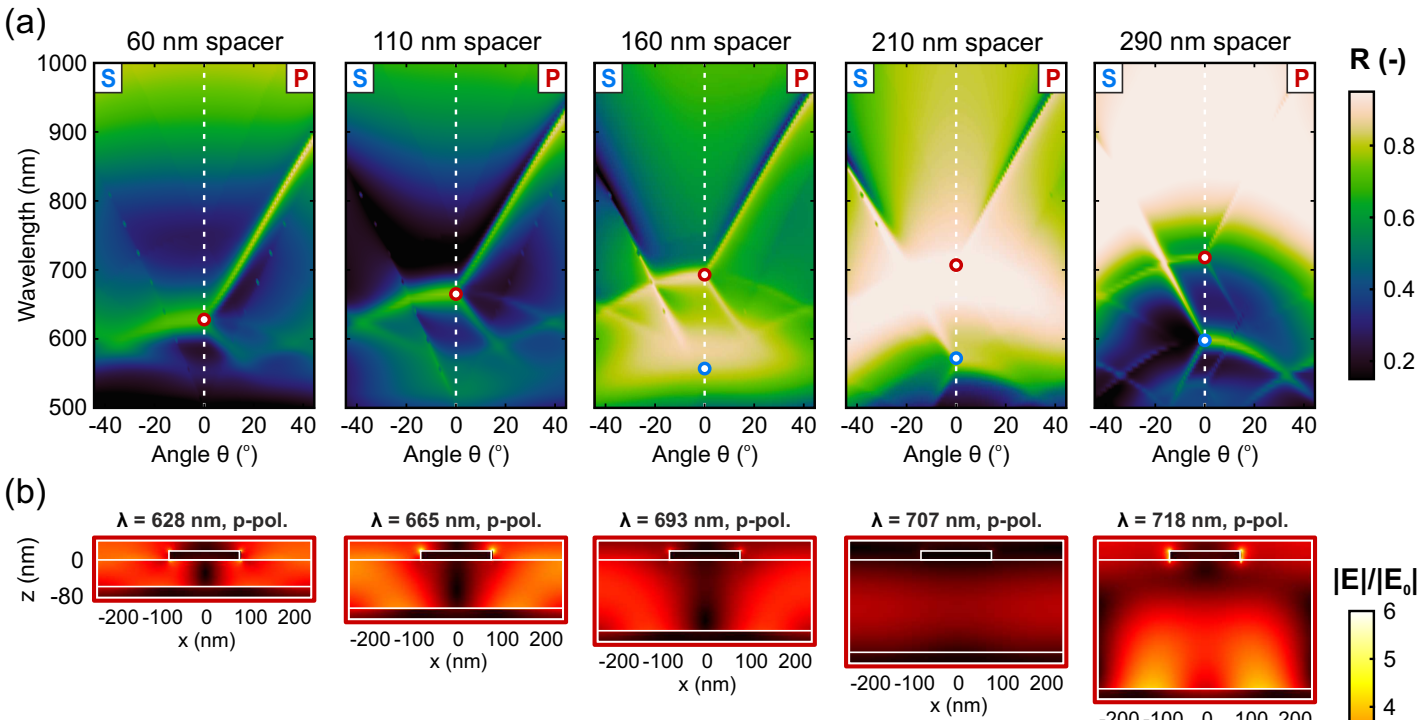

$\lambda=718 \mathrm{~nm}$,

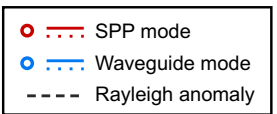

(c)

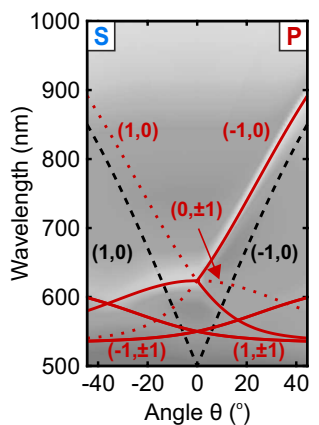

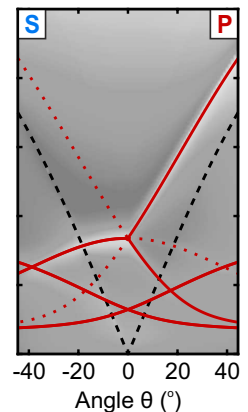

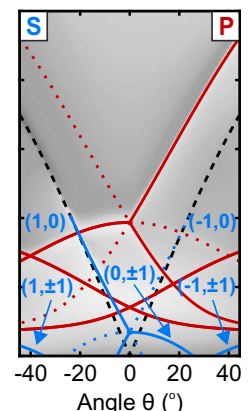

$\lambda=572 \mathrm{~nm}, \mathrm{p}-\mathrm{pol}$.
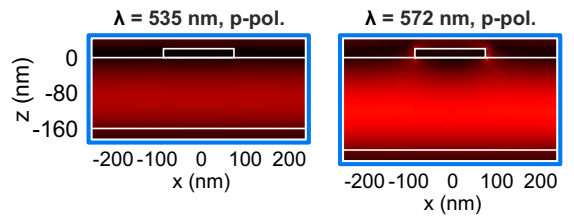

$\mathrm{x}(\mathrm{nm})$
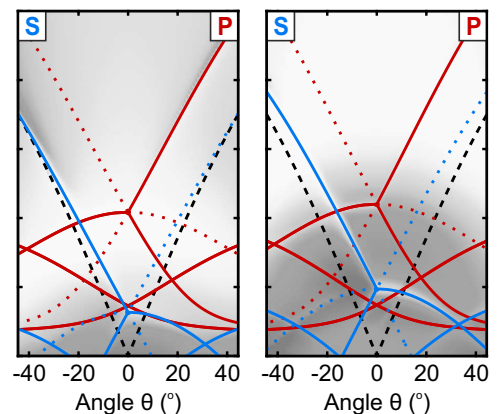

Figure S7: (a) Calculated reflectance vs. incident wavelength and angle for a Pd absorber structures with $P=500 \mathrm{~nm}, d_{\text {disk }}=160 \mathrm{~nm}$, for the five different fabricated spacer thicknesses. (b) Calculated field distributions at the spectral positions that are marked with red and blue dots in panel (a). The top row (red) reveals the excitation of a SPP mode at the interface between the gold mirror and the dielectric spacer, while the bottom row (blue) reveals the excitation of a waveguide mode inside the spacer. (c) Angle-dependent dispersion diagrams, calculated under the empty-lattice approximation [cf. equation S17]. Red and blue lines denote SPP and waveguide modes, respectively, and the black dashed lines indicate Rayleigh anomalies. The different diffraction orders are labeled with their corresponding values of $\left(m_{\mathrm{x}}, m_{\mathrm{y}}\right)$. For an easy comparison, a grayed-out version of the spectra from panel (a) is plotted in the background. Branches that cannot be excited due to symmetry reasons are displayed by dotted lines.

Although the SPP and the waveguide modes are eigenmodes of the homogeneous system, in this system, it would not be possible to excite them from the outside, since their dispersion relation is located outside the light cone of the surrounding medium. However, the situation differs as soon as any periodicity is introduced. In the following, we take into account the periodicity that is caused by the Pd nanodisks, but neglect a possible direct influence of the Pd nanodisks onto 
the propagation of the modes. In solid state physics and photonic crystal theory, this approach is known as the empty-lattice approximation [1,2]. Under this approximation, the dispersion relation of a mode in the periodic system is given by

$$
\omega_{\mathrm{m}}\left(k_{\|}\right)=\omega_{\mathrm{m}}\left[\sqrt{\left(k_{\mathrm{x}}+\frac{2 \pi}{p_{\mathrm{x}}} m_{\mathrm{x}}\right)^{2}+\left(k_{\mathrm{y}}+\frac{2 \pi}{p_{\mathrm{y}}} m_{\mathrm{y}}\right)^{2}}\right] .
$$

Here, $k_{\mathrm{x}}$ and $k_{\mathrm{y}}$ denote the $x$ and $y$ momentum components, respectively, which are imposed in the periodic system due to the momentum of incoming photons, $p_{\mathrm{x}}$ and $p_{\mathrm{y}}$ are the periodicities in the respective directions (in our case $p_{\mathrm{x}}=p_{\mathrm{y}}=P$ ), and $m_{\mathrm{x}}, m_{\mathrm{y}}=0, \pm 1, \pm 2, \ldots$ represent the possible diffraction orders. Mathematically, equation S16 describes a folding of $\omega_{\mathrm{m}}\left(k_{\|}\right)$into the first Brillouin zone. Due to this folding, formerly non-excitable modes of the homogeneous system that were located outside the light cone are now able to enter the light cone and can become excitable $[1,2]$.

Let us now take a deeper look at the excitation of the modes. In accordance with the measurements and the calculated spectra, we assume that the system is illuminated by a plane wave that impinges from top onto the structure. Let $\omega_{\text {inc }}$ be the frequency of the incident wave. The wave can excite a mode if $\omega_{\text {inc }} \approx \operatorname{Re}\left(\omega_{\mathrm{m}}\right)$. In our setup, we have $k_{\mathrm{x}}=\omega_{\text {inc }} / c \sin (\theta)$ and $k_{\mathrm{y}}=0$. Combining these relations with equation $\mathrm{S} 16$, and assuming that the function $\operatorname{Re}\left[\omega_{\mathrm{m}}\left(k_{\|}\right)\right]$is invertible, we can express the angle $\theta$, under which a mode can be excited, as a function of the incident frequency:

$$
\sin \theta=\frac{c}{\omega_{\text {inc }}}\left(-\frac{2 \pi}{p_{\mathrm{x}}} m_{\mathrm{x}} \pm \sqrt{k_{\|}^{2}\left(\omega_{\mathrm{inc}}\right)-\left(\frac{2 \pi}{p_{\mathrm{y}}} m_{\mathrm{y}}\right)^{2}}\right) .
$$

The numerically calculated dispersion relations of the SPP and the waveguide mode enter via the term $k_{\|}\left(\omega_{\text {inc }}\right)=k_{\|}\left[\operatorname{Re}\left(\omega_{\mathrm{m}}\right)\right]$. Besides these two modes, another feature can occur in periodic system, which is the so-called Rayleigh anomaly [7]. It can be described via equation S17 as well, by simply setting $k_{\|}\left(\omega_{\text {inc }}\right)=n \omega_{\text {inc }} / c$, where $n$ is the refractive index of the surrounding medium. In our case, the structure is covered by air on top, so that $n=1$.

The results of equation $\mathrm{S} 17$ with $P=500 \mathrm{~nm}$ are plotted in Figure S7(c). Red and blue lines correspond to SPP and waveguide modes, respectively, and black dashed lines indicate the Rayleigh anomalies. The different diffraction orders are labeled with their corresponding values of $\left(m_{\mathrm{x}}, m_{\mathrm{y}}\right)$. For an easy comparison, a grayed out version of the calculated spectra from Figure $\mathrm{S7}(\mathrm{a})$ is plotted in the background behind the curves. In consistency with the spectra, the left side of each plot (i.e., negative angles) refers to s polarization, while the right side (i.e. positive angles) refers to $p$ polarization. Simple symmetry considerations that take into account the TM polarization of the SPP mode and the TE polarization of the waveguide mode allow to rule out branches that cannot be excited by the incident $s$ and $p$ polarization, respectively. These branches are plotted with dotted instead of solid lines. As it can be seen, there is an excellent agreement between the numerically calculated spectra and the results of the empty-lattice approximation, which allows to clearly identify all observed spectral features. 


\section{References}

[1] Christ, A.; Zentgraf, T.; Tikhodeev, S. G.; Gippius, N. A.; Kuhl, J.; Giessen, H. Controlling the interaction between localized and delocalized surface plasmon modes: Experiment and numerical calculations. Phys. Rev. B 2006, 74, doi:10.1103/PhysRevB.74.155435.

[2] Christ, A.; Zentgraf, T.; Kuhl, J.; Tikhodeev, S. G.; Gippius, N. A.; Giessen, H. Optical properties of planar metallic photonic crystal structures: Experiment and theory. Phys. Rev. B 2004, 70, doi:doi:10.1103/PhysRevB.70.125113.

[3] Kichin, G.; Weiss, T.; Gao, H.; Henzie, J.; Odom, T. W.; Tikhodeev, S. G.; Giessen, H. Metaldielectric photonic crystal superlattice: $1 \mathrm{D}$ and $2 \mathrm{D}$ models and empty lattice approximation. Physica B: Condensed Matter 2012, 407, 4037-4042.

[4] Weiss, T.; Mesch, M.; Schäferling, M.; Giessen, H.; Langbein, W.; Muljarov, E. A. From Dark to Bright: First-Order Perturbation Theory with Analytical Mode Normalization for Plasmonic Nanoantenna Arrays Applied to Refractive Index Sensing. Phys. Rev. Lett. 2016, 116, doi:doi:10.1103/PhysRevLett.116.237401.

[5] Weiss, T.; Gippius, N. A.; Tikhodeev, S. G.; Granet, G.; Giessen, H. Derivation of plasmonic resonances in the Fourier modal method with adaptive spatial resolution and matched coordinates. J. Opt. Soc. Am. A 2011, 28, 238-244.

[6] Etchegoin, P. G.; Le Ru, E. C.; Meyer, M. An analytic model for the optical properties of gold. J. Chem. Phys. 2006, 125, doi:doi:10.1063/1.2360270.

[7] Weiss, T.; Schäferling, M.; Giessen, H.; Gippius, N. A.; Tikhodeev, S. G.; Langbein, W.; Muljarov, E. A. Analytical normalization of resonant states in photonic crystal slabs and periodic arrays of nanoantennas at oblique incidence. Phys. Rev. B 2017, 96, doi:doi:10.1063/1.2360270. 